\title{
High-throughput preparative separations from combinatorial libraries
}

\author{
K. Coleman
}

Anachem Ltd., 20 Charles St., Luton, Beds., LU2 OEB, United Kingdom

\begin{abstract}
Guidelines for high-throughput preparative separations with SFC methods are presented. Examples showing the use of generic modifier gradients and a single column can be successfully applied to cope with the large number of samples generated by combinatorial and medicinal chemists. The result is rapid achievement of high-purity analytes at the tens-of-milligram levels in a concentrated liquid form.
\end{abstract}

Keywords: SFC, Fast gradient, Combinatorial libraries, Drug discovery, Purification

\section{Introduction}

With the increased demands generated by pharmaceutical research for characterization and activity testing, a technique is required to speed up the isolation of substances at the tens-of-milligram levels from crude reactional mixtures synthesized as combinatorial libraries. Supercritical-fluid chromatography (SFC) has several advantages over high-performance liquid chromatography (HPLC) in the areas associated with chromatographic purification: 1) SFC is compatible with all solvents miscible with carbon dioxide, including aprotic solvents such as dimethyl sulfoxide (DMSO), obviating the need to re-solvate in a "friendly" solvent prior to purification. 2) SFC is a high-resolution technique; short separation times can be achieved without compromising resolution. 3) In general, the mass of sample loaded in SFC can be high and often higher than in HPLC [1-5]. 4) Liquid fractions are collected in a concentrated form, eliminating the time-consuming need to perform solvent removal.

This paper describes the instrumentation and the protocols used to achieve the goals outlined, and is supported by practical applications for diverse chemistries from a range of pharmaceutical industries.

\section{Instrumentation}

The chromatograph used is based on a conventional Gilson HPLC system (Villiers-le-Bel, France, and Middleton, WI,
USA) with high-pressure mixing of two solvents. Switching between HPLC and SFC is both convenient and routine. This system is a standard offer, assembled from the original Gilson SF3 configuration, and it can be tailored for more specific requirements $[1,2]$. The following components were utilized: pump A (306/10SC) fitted with a head-chiller unit (Anachem) for the delivery of liquid carbon dioxide. Pump B (306/10SC) for the delivery of organic modifiers and additives. High-pressure dynamic mixer (811C) with $1.5-\mathrm{ml}$ mixer volume. Auto-injector (215 with 1-ml dilutor syringe) for injection from microplates, vials or tubes, as well as fraction collection into vials and tubes. Rheodyne 7010 injection valve with $500-\mu l$ loop, mounted in a valve actuator (819). Oven unit (831) fitted with pressure-relief valve (7037) to facilitate aerosol formation and stream-splitting for mass detectors (MS, ELSD, etc.). Dual-wavelength UV detector (119) fitted with a high-pressure flow cell. Pressure regulator (821). Pump C (306/5SC) to ensure re-solvation of analytes after depressurization [2-4]. Valve actuator (819) fitted with Rheodyne 7010 switching valve to allow the Gilson 215 to perform as both an injector and a fraction collector $[4,6]$. System software (UniPoint).

For mass detection, an evaporative light-scattering detector (ELSD) Sedex 55 from Sedere (Alfortville, France) was also used as a second detector for some cases. To activate peak fraction-collection with the Gilson UniPoint software, any detector outputting an analog signal can be used. However, as with HPLC, when using mass detectors, the eluent flow must be split. With SFC, this is most conveniently achieved by utilizing a second outlet of the 7037 pressure-relief valve situated after the pressure-control valve, and then optimizing the split flow with a micro-splitter valve from Upchurch Scientific (Oak Harbor, WA, USA).

\section{Methods}

The following generic methods were implemented to rapidly isolate high-purity analytes, at the tens-of-milligrams levels in a concentrated liquid form from the large number of samples generated by combinatorial and medicinal chemists.

\section{Constant conditions}

In this study, the column was a cyano-propyl silica (Hypersil), $5 \mu \mathrm{m}, 12.5 \mathrm{~cm} \times 10$-mm ID. A diol column with similar retention characteristics can be used as an alternative 
phase. The mobile-phase flow rate was $8 \mathrm{ml} / \mathrm{min}$, the column temperature was $65{ }^{\circ} \mathrm{C}$ and the column outlet-pressure $14 \mathrm{MPa}$. Carbon dioxide was distillers-grade from BOC (Luton, UK). Polar modifier was either methanol or isopropanol, both of AR-grade from Lab-Scan (Dublin, Ireland) and both containing $0.1 \%$ trifluoroacetic acid (TFA) from Sigma. Injected samples were crude reactional mixtures, synthesized in various solvents such as DMSO, ethyl acetate and methanol, at concentrations generally higher than $100 \mathrm{mg} / \mathrm{ml}$. Typical preparative injection volumes were 150-250 $\mu \mathrm{l}$. For analytical purposes, the injection volume was $10 \mu \mathrm{l}$. The collection solvent, introduced between UV detector and pressure-regulation valve, was isopropanol from Lab-Scan, flowing at $1 \mathrm{ml} / \mathrm{min}$.

\section{Generic composition gradients}

The following two approaches were successfully implemented, both using generic gradient programs for the modifier composition:

1) When the highest possible sample-number throughput was required, fast gradient elution was obtained with the 7-min cycles defined in table I, and applied to all samples. For the types of chemistries studied, this approach showed a success rate higher than $90 \%$, with peak purity higher than $85 \%$ (see Results and discussion below).

2) When time allowed, or a greater success rate was desired, two different runs were successively implemented. First, a preliminary scouting run was carried out, using analytical loading $(10 \mu \mathrm{l})$ and the 20 -min cycles defined in table II, applied to all samples to locate the elution time of the compounds of interest. Then, a specific "rapid" gradient was run, using preparative loading (150-250 $\mu \mathrm{l})$ and, according to the elution time obtained in the previous run, one of the three 8-min cycles defined in table III to allocate an appropriate gradient $(2 \mathrm{a}, 2 \mathrm{~b}$ or $2 \mathrm{c})$ to each sample individually. From the results of the analytical scouting run, this appropriate preparative gradient profile was selected with the following criteria:

a) If the analytes of interest eluted within five minutes, gradient $2 \mathrm{a}$ of table III was chosen to increase retention and resolution. This gradient covers the initial ramp 1 of the scout gradient of table II. Alternatively, a more polar column (e.g. bare silica) could be used.

b) If the analytes eluted between 5 and 14 min, gradient $2 b$ (Tab. III) corresponding to ramp 2 (Tab. II), was used.

c) If the analytes were eluted after 14 min, gradient $2 \mathrm{c}$ (Tab. III) corresponding to ramp 3 (Tab. II) was performed.

A much more time-consuming alternative would consist of tuning each preparative gradient to maximize separation and minimize cycle time for each sample individually.
Table I. Generic "rapid" gradient 1 , using a single ramp and 7-min cycles.

\begin{tabular}{cc}
\hline Time $(\mathrm{min})$ & Modifier (\%) \\
\hline 0 & 1 \\
0.5 & 1 \\
4.5 & 45 \\
5.5 & 45 \\
5.6 & 1 \\
7 & 1 \\
\hline
\end{tabular}

Table II. Generic "scout" gradient 2, using three ramps and 20 -min cycles.

\begin{tabular}{cc}
\hline Time $(\mathrm{min})$ & Modifier $(\%)$ \\
\hline 0 & 1 \\
0.5 & 1 \\
3 & $10($ ramp 1$)$ \\
13 & $25($ ramp 2$)$ \\
18 & $45($ ramp 3$)$ \\
18.5 & 1 \\
20 & 1 \\
\hline
\end{tabular}

Table III. Generic "rapid" gradients 2a, $2 \mathrm{~b}$ and 2c, using two or three ramps and 8-min cycles.

\begin{tabular}{lccc}
\hline Time (min) & $\begin{array}{c}\text { Gradient 2a } \\
\text { Modifier (\%) }\end{array}$ & $\begin{array}{c}\text { Gradient 2b } \\
\text { Modifier (\%) }\end{array}$ & $\begin{array}{c}\text { Gradient 2c } \\
\text { Modifier (\%) }\end{array}$ \\
\hline 0 & 1 & 1 & 1 \\
0.5 & $(1)$ & 1 & 1 \\
1 & 1 & 7.5 & 22.5 \\
5 & 10 & 27.5 & 45 \\
6 & 10 & 27.5 & $(45)$ \\
6.1 & 45 & 45 & $(45)$ \\
6.5 & 45 & 45 & 45 \\
6.6 & 1 & 1 & 1 \\
8 & 1 & 1 & 1 \\
\hline
\end{tabular}

\section{Results and discussion}

Figure 1 shows the results obtained for three unrelated reactional mixtures of combinatorial chemistry analyzed by both the "rapid" and "scout" gradient conditions. Employing the "rapid" gradient, mixtures A and B show sufficient resolution of the main component for preparative isolation, but mixture $\mathrm{C}$ would better be purified using gradient $2 \mathrm{~b}$. 

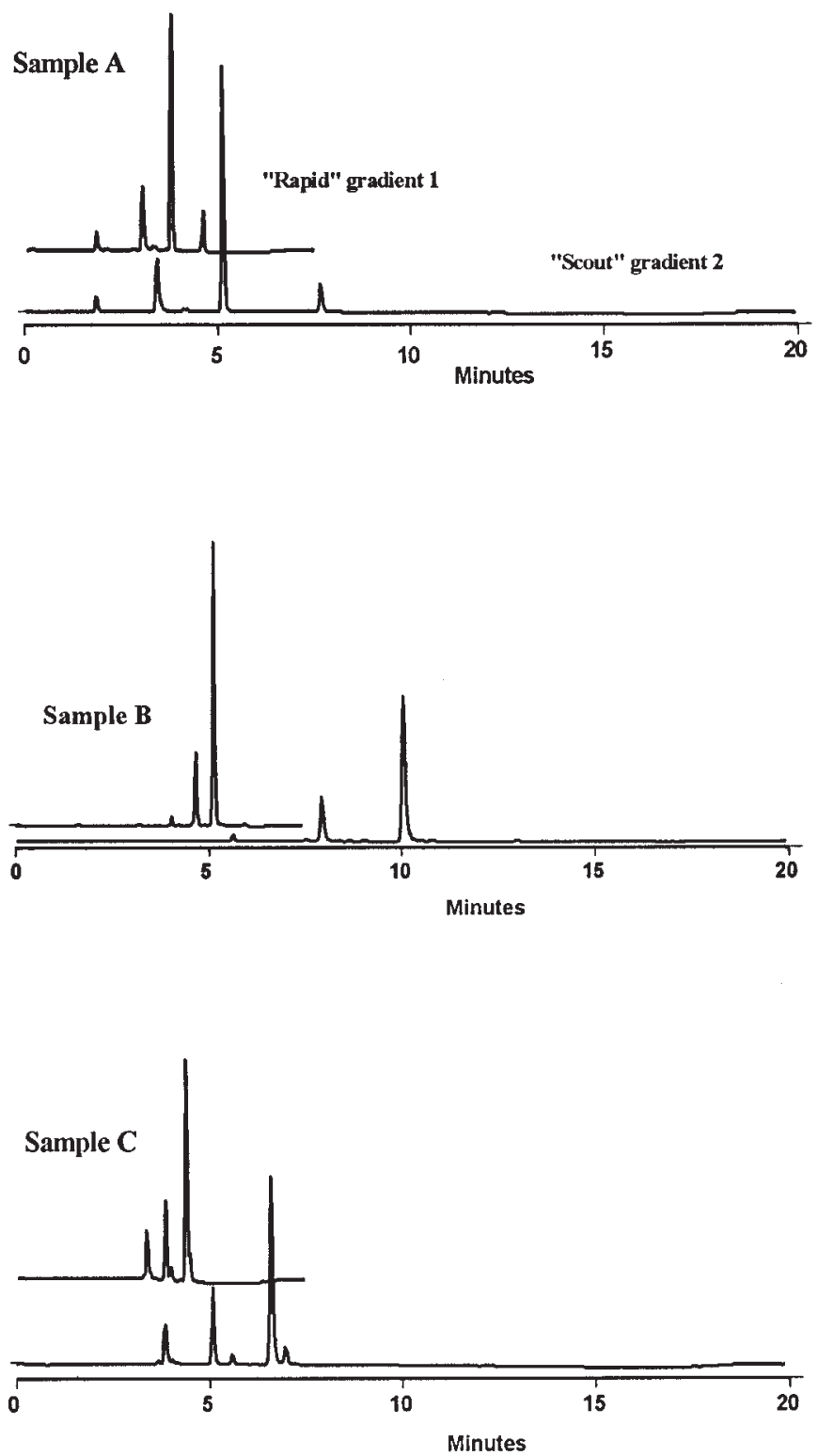

Figure 1. Comparison of "rapid" gradient 1 with "scout" gradient 2. Samples: three unrelated synthesis mixtures, A, B and $C$. Mobile phase: carbon dioxide and methanol containing $0.1 \%$ TFA, see tables I and II for gradient conditions. Injection volume: $10 \mu \mathrm{l}$. Detection: UV at $254 \mathrm{~nm}$.

\section{Sample loading}

Since the mass loadability in SFC is often higher than with HPLC, and is compatible with organic solvents miscible with carbon dioxide (including polar and aprotic solvents), crude synthesis reactional mixtures can preparatively be injected directly. This was the case for all samples (A-G) in this study. The volume that was injected, before peak distortion was observed, depended on the sample solvent and on the analytes retention. It was typically $150-250 \mu 1$ on the

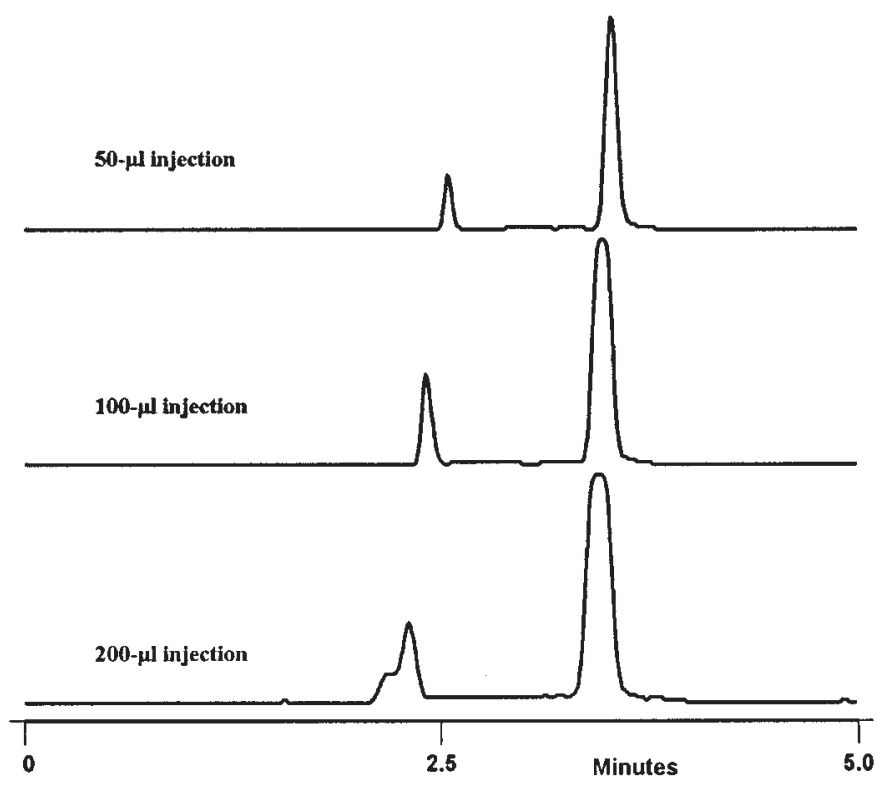

Figure 2a. Effect of increasing the sample volume. Sample: reactional mixture D synthesized in DMSO. Mobile phase: carbon dioxide and isopropanol containing $0.1 \%$ TFA, gradient 1 (see table I). Injection volumes: 50, 100 and $200 \mu$ l. Detection: UV at $230 \mathrm{~nm}$.

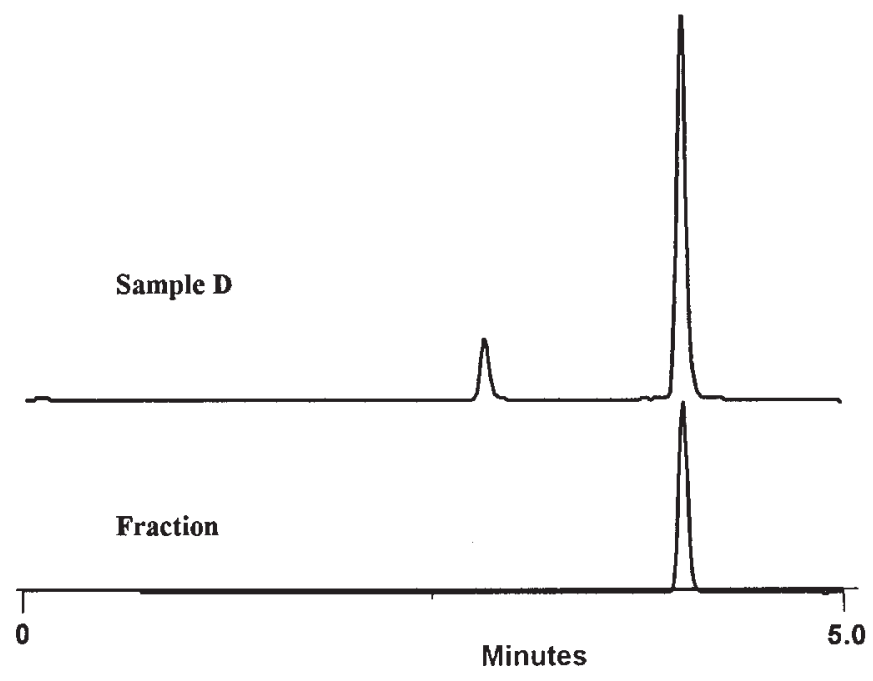

Figure 2b. Fraction analysis. Conditions: gradient 1 (see table I) and detection at $230 \mathrm{~nm}$. Fraction purity: $98 \%$ (from $86 \%$ in the original sample D).

10-mm-ID column used. Furthermore, this volume was more dependent on the sample solvent than on the sample concentration. For instance, for the least-polar analytes at high concentration in DMSO, the injection volume had to be decreased in order maintain the desired cycle time, that 


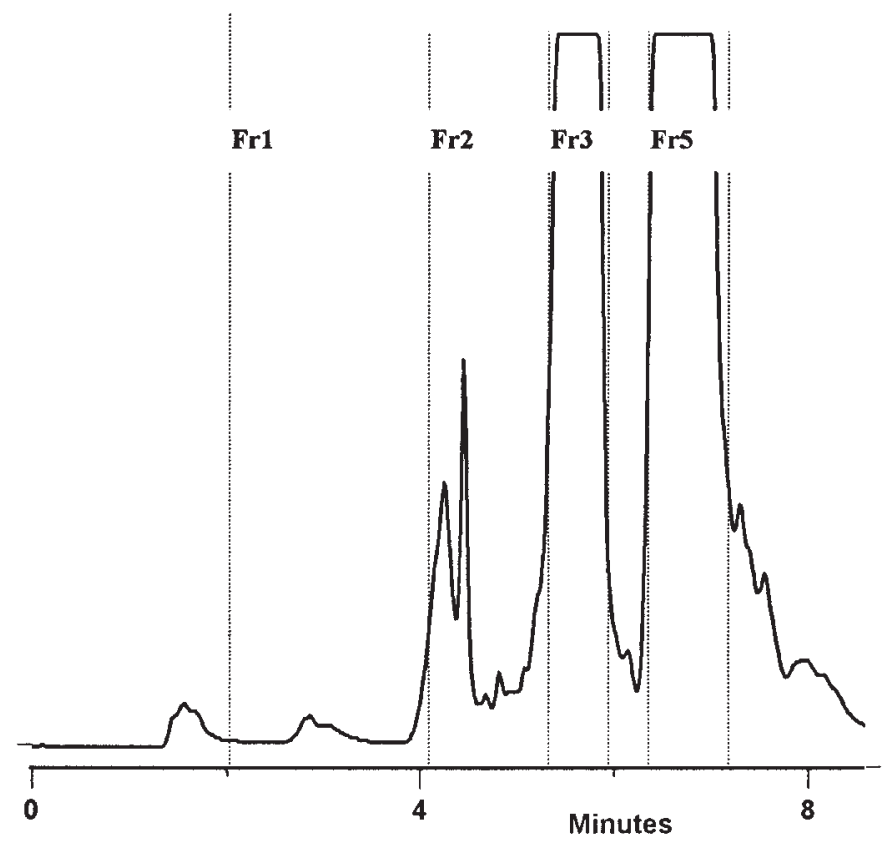

Figure 3a. Threshold-activated fraction collection. Sample: reactional mixture E synthesized in DMSO. Mobile phase: carbon dioxide and methanol containing $0.1 \%$ TFA, gradient $2 \mathrm{~b}$ (see table III). Injection volume: $200 \mu \mathrm{l}$. Detection: UV at $254 \mathrm{~nm}$.

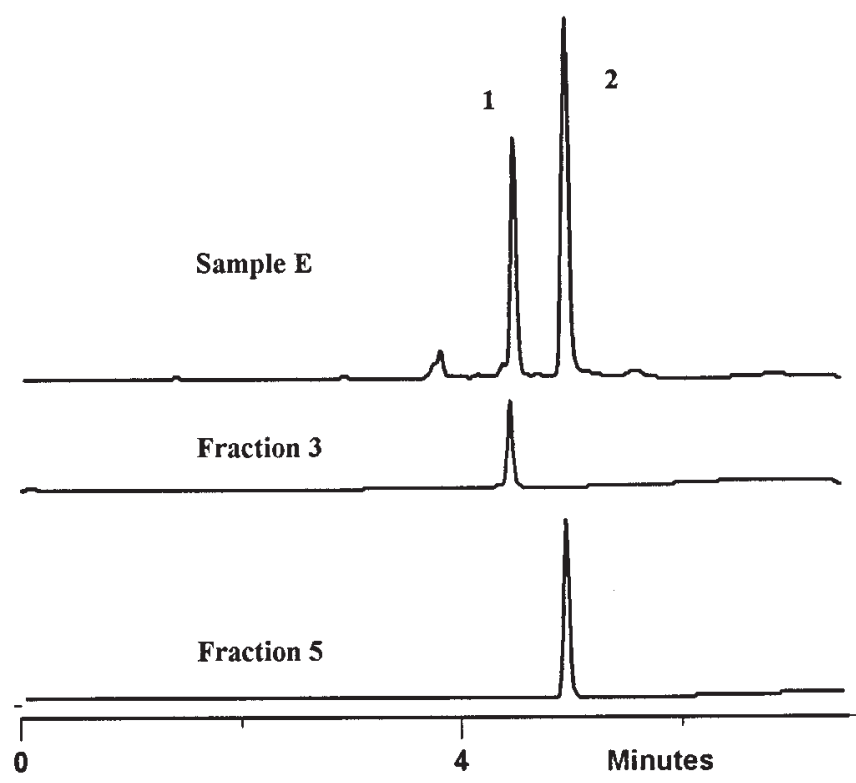

Figure 3b. Fraction analysis. Conditions: gradient 1 (see table I) and detection at $254 \mathrm{~nm}$. Purity of fraction 3 (peak 1): > $97 \%$ (from $30 \%$ in the original sample E). Purity of fraction 5 (peak 2): > $99 \%$ (from $57 \%$ in the original sample E).

otherwise would have required a longer column regeneration time.

Figure $2 \mathrm{a}$ shows the results of increasing the injection volume of a synthesis mixture in DMSO from 50 to $200 \mu$ l.

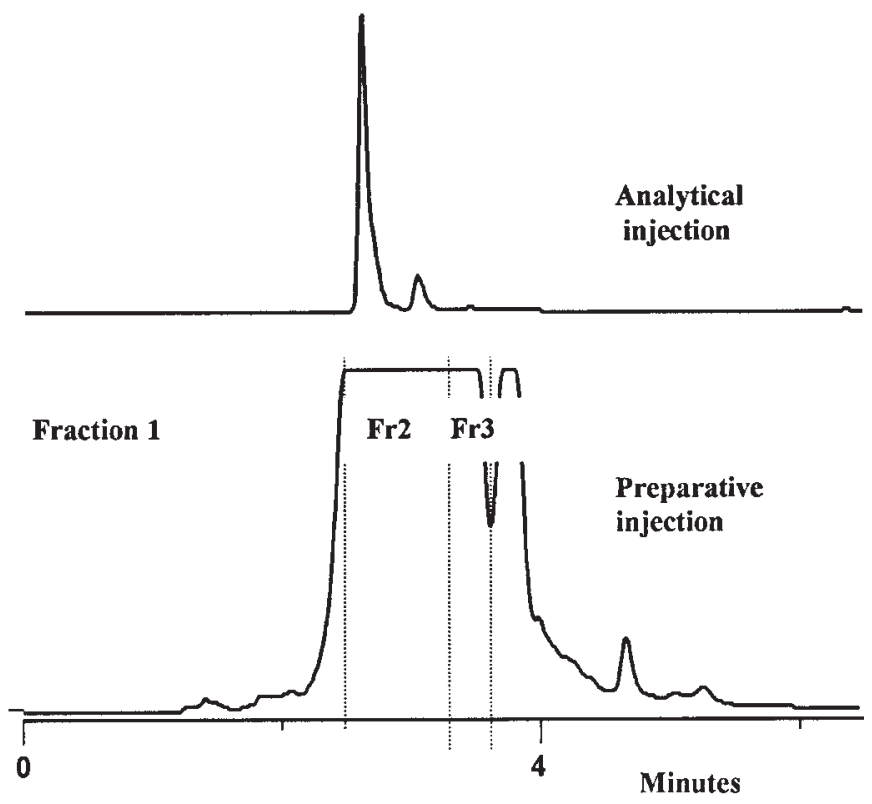

Figure 4a. Effect of employing the "time-per-tube" collection mode. Sample: reactional mixture $F$ synthesized in ethyl acetate. Mobile phase: carbon dioxide and isopropanol containing $0.1 \%$ TFA, gradient 1 (see table I). Injection volumes: $10 \mu \mathrm{l}$ (analytical) and $150 \mu \mathrm{l}$ (preparative). Detection: UV at $230 \mathrm{~nm}$.

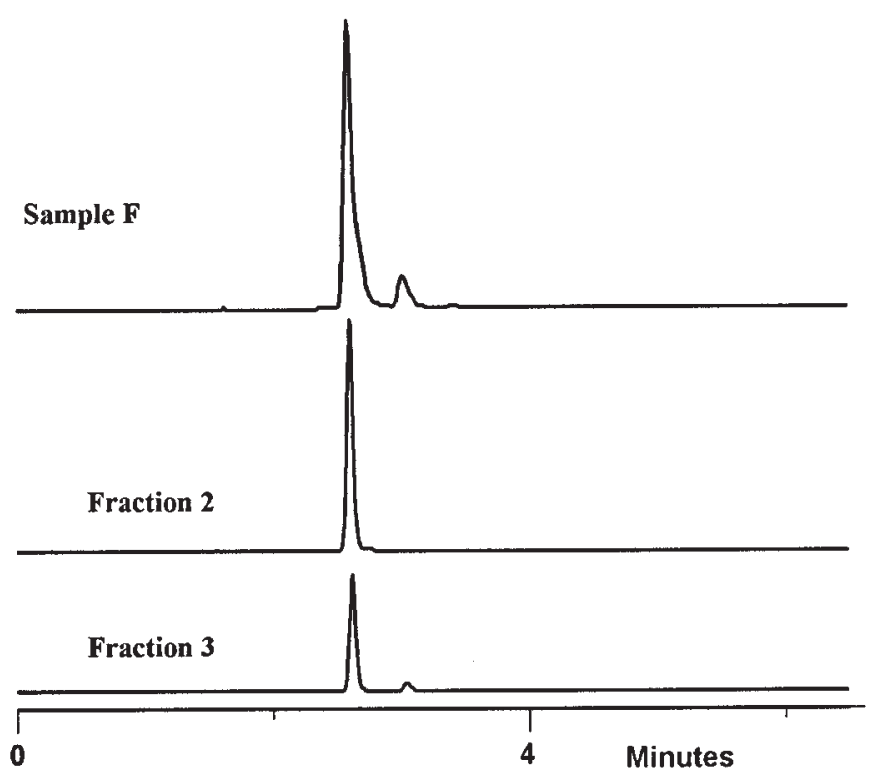

Figure 4b. Fraction analysis. Conditions: gradient 1 (see table I) and detection at $230 \mathrm{~nm}$. Fraction purity: $98 \%$ for fraction 1 and $92 \%$ for fraction 3 (from $85 \%$ in the original sample F).

For $200 \mu 1$, peak splitting is observed for the less polar component. Figure $2 b$ shows the fraction analysis after preparative SFC. 


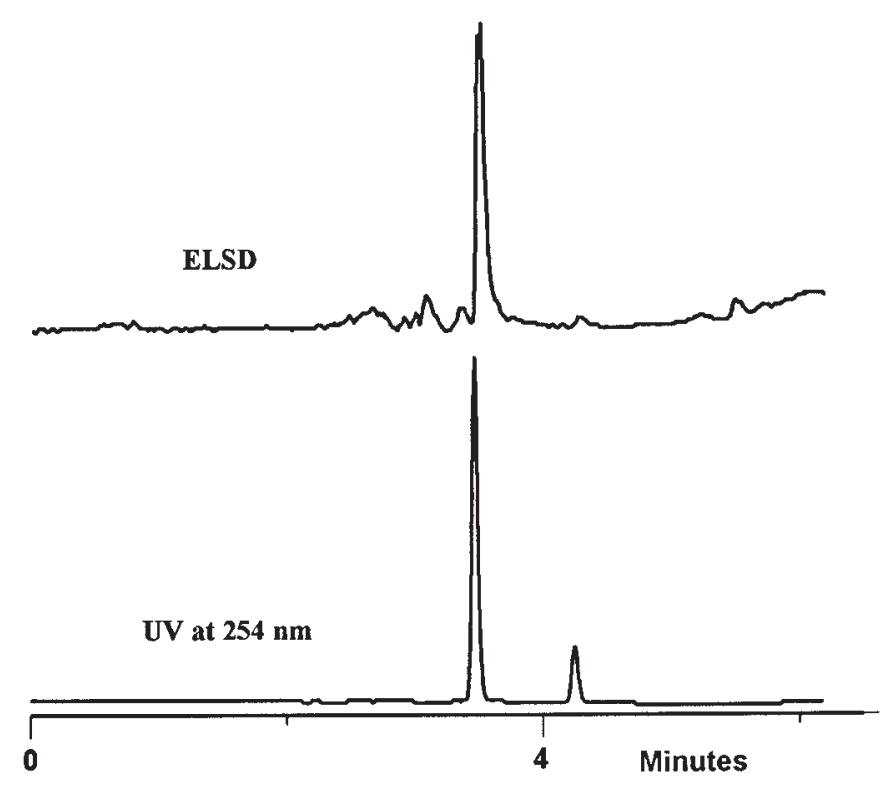

Figure 5. Use of two dectectors. Sample: reactional mixture G synthetized in methanol. Mobile phase: carbon dioxide and methanol containing $0.1 \%$ TFA, gradient 1 (see table I). Injection volume: $10 \mu \mathrm{l}$. Detection: UV at $254 \mathrm{~nm}$ (total flow) and ELSD (split flow).

\section{Fraction collection and detection}

As with HPLC, fraction collection is typically activated by the detector response exceeding a set threshold. With combinatorial synthesis there may be several major components, therefore to minimize the number of fractions collected per sample, it is recommended the threshold be set as high as possible and that a selective mode of detection be employed. To avoid missing the component of interest, it is also recommended to practice "non-peak" collection in combination with peak collection. Figure 3 a shows a synthesis mixture containing two major components. By using a detector response to activate fraction collection both components are collected (fractions 3 and 5). Figure $3 b$ shows the results after preparative SFC.
When scaling up to preparative loading, it is probable that closely eluting components will merge, particularly if the "rapid" gradient conditions are employed, it is therefore recommended to limit the peak collection time-per-tube to maximize the chances of collecting pure components. Figure 4a shows the peak collection time limited to $0.5 \mathrm{~min}$ per tube, and figure $4 \mathrm{~b}$ shows the resulting fraction purities.

Last, figure 5 shows the system interfaced to the Sedex 55 ELSD, with a split flow to the detector of less than $1 \%$ of the total flow.

\section{Conclusion}

As has been demonstrated, preparative SFC, when applied to the field of combinatorial chemistry, is a means of rapid routine purification. Analytical and preparative SFC has previously been reported as the preferred process in many other areas of pharmaceutical drug discovery. The LC/SFC system described can be applied to all these fields, giving the analyst and chemist increased confidence for meeting their analytical and preparative requirements.

\section{References}

1. Coleman, K.; Vérillon, F. Laboratory-scale preparative chromatography enhanced by fluids containing carbon dioxide under automated pressure control, Proceedings of the $3^{\text {rd }}$ International Symposium on Supercritical Fluids: Strasbourg, France, 1994; pp 415-420.

2. Vérillon, F.; Coleman, K., Analytical and preparative carbon dioxide chromatography with automated pressure control and different detectors, In: Anton, K. and Berger, C., Supercritical fluid chromatography with packed columns: techniques and applications; Marcel Dekker Inc.: New York, NY, 1997; ch. 3, pp 59-95.

3. Ashraf-Khorassani, M.; Gandee, M. B.; Combs, M. T.; Taylor, L.; Frey, C. R. J. Chromatography. Sci. 1997, 35, 593-597.

4. Coleman, K.; Boutant, R.; Vérillon, F. Isolation and Purification, 1999, 3, 9-19.

5. Vérillon F., For preparative work, the sample mass throughput is higher in SFC than in LC?, Gilson Product Information 104, 25 June 1999.

6. Vérillon, F.; Pichon, B.; Qian, F. Am. Lab. 1987, 19, 88-95. 